Canadian Oncology

Nursing Journal

Revue canadienne

de soins infirmiers

en oncologie

Volume 28, Issue 1 • Winter 2018

elSSN: 2368-8076 


\title{
Empowering patients and caregivers with knowledge: The development of a nurse-led gynecologic oncology chemotherapy education class
}

\author{
by Nazlin Jivraj, Lisa Ould Gallagher, Janet Papadakos, Nazek Abdelmutti, Aileen Trang and Sarah E. Ferguson
}

\begin{abstract}
At a Canadian cancer centre, rising patient volumes made it diffcult to provide quality chemotherapy education to patients and families in the clinical setting. The gynecology oncology site identified several barriers to the provision of timely and comprehensive teaching. These barriers included receiving education after learning of a cancer diagnosis, the efficacy of written information, time constraints nurses experienced, and absence of standardized side effect management. This prompted an interdisciplinary team to review current teaching practices and engage the Patient Education Program to collaboratively develop strategies to overcome these challenges. This paper describes the development of a nurse-led chemotherapy education class tailored to patients with gynecologic cancers and focused on common chemotherapy treatment protocols. The purpose of the class was to help patients and caregivers know what to expect during their chemotherapy routine, lower anxiety, and to equip them with knowledge and skills to manage side effects of treatment.
\end{abstract}

\section{INTRODUCTION}

$\mathrm{T}$ The Canadian Cancer Society (2015) reported an estimated 196,900 people in Canada were diagnosed with cancer in 2015. Of this number, approximately 10,508 had a gynecologic cancer. These incidence rates have been noted at our cancer

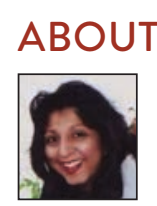

THE AUTHORS

Nazlin Jivraj, RN, BScN, CON(C), Specialized Oncology Nurse, Gynecology Oncology Ambulatory Care, Princess Margaret

Cancer Centre, Toronto, ON. Email: nazlin.jivraj@uhn.ca

(Corresponding author)

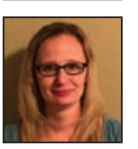

Lisa Ould Gallagher, RN, CON(C): Specialized Oncology Nurse, Gynecology Oncology Ambulatory Care, Princess Margaret Cancer Centre, Toronto, ON. Email: lisa.gallagher@uhn.ca

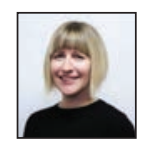

Janet Papadakos, MEd, PhD, Co-Director, Cancer Health Literacy Research Centre, Senior Manager, Cancer Education Research, Princess Margaret Cancer Centre; Provincial Head, Patient Education, Cancer Care Ontario. Toronto, ON. Email: Janet.Papadakos@uhnresearch.ca

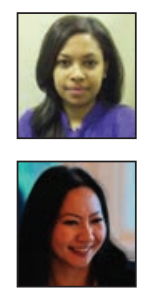

Nazek Abdelmutti, MSc (Htlh Communication), Manager, Cancer Strategy Stewardship, Princess Margaret Cancer Centre, Toronto, ON.

Email: Nazek.abdelmutti@uhnresearch.ca

Aileen Trang, MSc: Division of Medical Oncology and Hematology, Clinical Trials Group, Princess Margaret Cancer Centre, Toronto, ON. Email: aileen.trang@uhn.ca

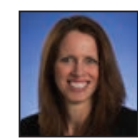

Sarah E. Ferguson, MD, FRCSC: Division of Gynecologic Oncology, University Health Network, Department of Obstetrics and Gynecology, University of Toronto, ON.

Email: sarah.ferguson@uhn.ca

DOI: $10.5737 / 2368807628147$ centre. In 2011, the cancer centre saw nearly 700 patients newly diagnosed with gynecologic cancers compared to 2001 when an estimated 494 patients were seen with the same malignancies (Cancer Registry, 2016). These rising patient volumes challenged the ability of oncology nurses to engage and educate patients in the outpatient setting, as time is limited and the education demands are high.

In addition, the chemotherapy treatment protocols for the management of gynecological malignancies include chemotherapy alone or in combination with radiation. These treatments are complex and require significant demands from patients including adherence with protocols. Adherence is difficult to achieve without comprehensive education and training. Certified oncology nurses are equipped with expertise in providing patients and caregivers with the necessary knowledge (Canadian Association of Nurses in Oncology [CANO], 2006) given their practice is guided by the standards set by the Canadian Association of Nurses in Oncology (CANO). The CANO practice standards mandate that specialized oncology nurses are responsible for teaching patients and caregivers about treatment protocols, side effect management, and coping strategies (CANO, 2006).

Nurses in the gynecology clinics experienced challenges when trying to provide comprehensive patient education due to time constraints and compounded by high levels of patient anxiety related to the recent diagnosis and fear associated with initiating chemotherapy treatment (Askren, 2013; Dalby et al., 2013; Sheldon, 2008). This was evident by an increasing number of patient phone calls to the oncology nurses for information about management of side effects, clarity on anti-emetic use, chemotherapy routines, or when to visit the emergency department. The growing number of patient concerns, combined with the rising patient volumes, directly impacted the nurse's ability to engage patients in comprehensive patient education and management. As a result of these factors, the gynecology oncology nurse-led chemotherapy education class was initiated. In this paper, the authors discuss the development of the class, the class curriculum, and evaluation.

\section{BACKGROUND}

Much published literature has addressed the challenges related to providing treatment and treatment-related education to cancer patients (Askren, 2013; Chelf et al., 2001; Dalby et al., 2013; Malone, 2007; Portz \& Johnston, 2014; Williams $\&$ Schreier, 2004). These challenges include the timing of patient education at disclosure of diagnosis (Malone, 2007; Williams \& Schreier, 2004), the effectiveness of written material (Williams \& Schreier, 2004; Chelf et al. 2001; Dalby et al., 
2013), time constraints faced by nurses (Askren, 2013) and the lack of standardized approaches to side effect management (Portz \& Johnston, 2014). The challenges outlined in the literature reflect those faced by nurses at our cancer centre.

Malone (2007) and Williams and Schreier (2004) reported that when patients learn of their cancer diagnosis and that their treatment includes chemotherapy, they experience anxiety. They can easily become overwhelmed and unable to comprehend or retain information related to chemotherapy side effects and routine. When patients are unable to comprehend or retain information about chemotherapy management, they may make unnecessary trips to the Emergency Departments (Mann, 2011); they may fail to recognize potentially dangerous symptoms and present to Emergency Departments after symptoms are exacerbated (Schofield, 2008; D'Amato, 2008). In turn, this can interrupt the treatment plan and its effectiveness (D’Amato, 2008).

At our cancer centre, patients in outpatient clinics are routinely told about their diagnosis and treatment plan either following gynecologic surgery or a diagnostic procedure. As a result, oncology nurses observed that many patients were unable to retain information about starting chemotherapy. Patients would call the nurse several times following their initial dose of chemotherapy in order to clarify management of side effects, medications and the chemotherapy schedule.

Compounding the significant impact of anxiety and feeling overwhelmed, and the patient's ability to comprehend cancer information, patient education materials are often written at grade levels far above the average patient's functional literacy skills. (Portz \& Johnston, 2014; Williams \& Schreier, 2004; Chelf et al., 2001; Dalby et al., 2013). Routinely, nurses utilized chemotherapy information sheets to review the side effects of treatment with the patient and caregiver. However, according to Williams and Schreier (2004), such information is often not at a grade level appropriate for many people to comprehend. Several authors note that these materials should be written in plain language, below or at grade six reading level, since patients present with varied capacity for reading (Chelf et al., 2001; Dalby et al., 2013; Doak, Doak \& Root, 1996).

Another significant challenge to the provision of chemotherapy patient education is time. A survey study conducted by Askren (2013) found that nurses were struggling with multiple demands on their time, which presented significant challenges to nurse-patient teaching. Askren (2013) wrote that nurses reported not being able to complete patient education due to challenges such as heavy patient loads, needing more than an hour to teach chemotherapy-naive patients, and patients feeling overwhelmed with too much information. This prompted a further assessment of patients and caregivers, asking them if they felt the information provided was adequate and helpful. They reported that their education for symptom management was not sufficient (Askren, 2013). Without sufficient education, the result can be poor symptom management, high levels of anxiety, and an inability to cope (McCaughan \& McKenna, 2007).

A dearth of time for teaching was a familiar challenge the gynecology oncology nurses encountered in our outpatient setting. Nurses experienced time constraints when attempting to teach patients and caregivers about chemotherapy, as it would conflict with other nursing responsibilities in the clinic. These time pressures did not allow for a productive learning environment, especially when patients were already distressed. Often, nurses would be providing education for two or three chemotherapy-naive patients while managing a clinic of 25 patients within a three-and-a-half-hour timeframe.

The gynecology oncology nurses also identified that management of some side effects was not standardized. This meant each nurse had his or her own methods for managing side effects. This created confusion for patients when they called the nurses for assistance. The importance of a standardized approach to patient education for initial chemotherapy teaching was also reflected in the work of Dalby et al. (2013). The Dalby team set out to improve patient care and staff satisfaction through the use of a standardized checklist, education materials and teaching methods.

To address these issues, the gynecology oncology nurses collaborated with the Patient Education Program to explore opportunities to leverage existing in-house patient education programs. One of the programs we explored was a general chemotherapy education class for patients and caregivers. This class content was broad, as it was developed for patients with various malignancies receiving any type of chemotherapy regimen. In 2011, an evaluation of this class showed it lowered anxiety levels of patients, but increased anxiety among caregivers (Papadakos, 2010). In responding to the open-ended questions in the evaluation, patients and caregivers shared that the class curricula were too broad and it would be better to attend a class that was tailored to the disease site and specific chemotherapy regimens. Patients and caregivers found the information provided in the class was overwhelming and often not applicable to them (Papadakos, 2010). As a result, the decision was made to develop a gynecologic cancer specific chemotherapy class.

\section{Development of a tailored class}

To support the development of a tailored gynecology chemotherapy class, the nurses obtained data from the Oncology Patient Information System (OPIS) at the cancer centre. The numbers from 2010 revealed approximately 250 patients received a new treatment regimen (OPIS, 2010). These results prompted further analysis in 2011 where each gynecology oncology nurse collected metrics for their own individual practice for a one-month period. The nurses collected information on three metrics: the number of new chemotherapy teaching sessions with patients; the amount of time spent on patient teaching; and the number of phone calls related to chemotherapy management including side effects, medications, and schedules.

The findings for the month of April 2011 showed 37 patients started a new treatment protocol. Based on tracking the amount of time nurses spent on patient teaching, it was determined that the average amount of time spent with each patient was 20 minutes. The workload was divided among one part-time and six full-time gynecology oncology nurses. If language was a barrier and an interpreter was used, the chemotherapy education took significantly longer. 
Twenty minutes per patient was seen to be insufficient based on the number of phone calls from patients asking for clarification. Patient call logs were reviewed and revealed a large number of phone calls each day seeking clarification about what had been taught during the 20-minute session. For the month of April 2011, nurses obtained metrics for the following type of calls: side effects, medications and schedules. There were 168 calls; 139 were related to symptom management; six were questions about how to take medications, and 23 were queries about appointment schedules. The nature of the phone calls indicated that most of the patients did not remember the information provided about symptom management, medications, and understanding of appointment schedule. Almost $83 \%$ of calls were associated with symptom control. Specifically, patients were calling with questions and concerns about nausea, vomiting, constipation, bony pain, fevers, and neuropathy.

These findings were presented to the gynecology oncology site group together with a proposal for the development of a gynecologic oncology chemotherapy education class to help increase patient and caregiver knowledge and to decrease the number of phone calls to nursing. The nursing and medical group agreed on the common chemotherapy protocols to be taught, as well as standardizing the management of side effects of treatment. The consensus was to start with the three most common protocols for the gynecology oncology patient population: Carboplatin, Paclitaxel and Cisplatin. Physicians or nurses would identify all chemotherapy-naive patients who would attend the class one week before starting treatment. Patients were booked into the chemotherapy education class as a scheduled mandatory appointment before initiating chemotherapy.

It was decided the class would be taught by a certified oncology nurse with expertise in gynecology oncology, in a quiet group setting for both patients and caregivers (Keller, 2006). The curriculum was developed to focus on four learning goals: what to expect during visits, how to manage side effects, how to cope with emotions, and how family and friends can help.

In addition to the one-hour class, chemotherapy protocol guides were developed. These guides are written at a grade six level to augment the information taught in the class. For instance, the guide contains information on how to take antiemetic medications and when to call the oncology nurse or go to the emergency department (Malone, 2007; Dalby et al., 2013). The gynecology oncology nurses collaborated with the oncologists and with the Patient Education Program in the development of these resources and the slide presentation for the class.

\section{RESULTS}

Between January 2012 and December 2015, 538 patients and 506 caregivers attended the class. In addition, various staff members such as nurses, patient flow coordinators, pharmacists, social workers, nurse managers, and medical fellows also periodically joined the class. Although metrics were not kept, nurses have observed significant decreases in the number of calls related to the management of chemotherapy medications, side effects, and appointments since the initiation of the class. We believe this illustrates that having dedicated time to educate patients and caregivers about their treatment, as a separate clinic visit, has improved outpatient clinic time management and increased personalized care for these patients. This is demonstrated by evaluations completed at the end of each class by patients and caregivers. Various testimonials from patients endorsed the need for this nurse-led project:

- "I think the class is a very good idea instead of just handouts... Knowing what to expect is more comforting than just not knowing. It made me feel more secure knowing that I will have all the help and guidance I will need through my journey."

- "This information provided was very helpful and helped take the 'unknown' and fear away."

- "Relax and non-threatening environment. Educator made me feel comfortable to ask questions."

Caregiver testimonials supported the patient feedback:

- "The educators were very informative and answered questions. It did help me, as a family member, answer some questions that I was concerned about, I am glad that I attended this session."

- "I think it is very important to bring a caregiver to take notes for the patient. A lot of information to take in. Presenter was very knowledgeable and well-spoken."

- "I think it would be very beneficial for the patient to have a family (that lives with them) to attend as well."

An important lesson from these evaluations was the value of tailoring the information to the specific patient population. This meant we needed to further develop the curriculum so that each of the chemotherapy regimens were addressed separately. Initially, all patients receiving chemotherapy were in the same class, regardless of their regimens. For example, the nurse would teach patients receiving Carboplatin and Paclitaxel or concurrent Cisplatin and radiotherapy, in the same class. Patients reported this method was confusing for them. Therefore, the nurses decided to hold separate classes for patients receiving each treatment regimen. Currently there are three one-hour nurse-led chemotherapy education classes held once a week:

- Intravenous Paclitaxel and Carboplatin chemotherapy (weekly)

- Intraperitoneal Cisplatin and Paclitaxel chemotherapy (biweekly)

- Concurrent Radiation and Cisplatin chemotherapy (biweekly)

The gynecology oncology site group has made the class a standard of care for all chemotherapy naive patients. In addition, newly hired staff and nursing students must attend the class as this provides an opportunity for staff to understand what the patients are learning and subsequently standardizes patient care. The gynecology chemotherapy education class has set a precedent for other site groups at the cancer centre to standardize their approach in teaching their patient populations. 


\section{NEW INITIATIVES}

With the overwhelming success of the class, there are several new initiatives being considered for further development. Many of these initiatives are based on feedback received from patients and caregivers. The following projects are under consideration:

- Develop a video series to highlight key components of chemotherapy self-care

- Make all materials available on the Internet for patients and caregivers to access

- Translate all resources into the priority languages of the cancer centre

- Develop site chemotherapy protocol guides for all of gynecology

- Develop a resource package for caregivers, with a focus on "Caregiver Stress"

- Develop an e-learning version of the class to standardize teaching and to help patients who come from out of town

- Develop an e-learning version of the class for staff and trainees to help standardize patient and caregiver teaching

\section{REFERENCES}

Askren, H. (2013). Patient binders: Creating a tool that helps patients and nurses. Retrieved from http://www. oncologynurseadvisor.com/survivorship/patientbinders-creating-a-tool-that-helps-patients-and-nurses / article/310253/

Canadian Association of Nurses in Oncology/Association canadienne des infirmières en oncologie (CANO \ACIO). (2006). Practice Standards and Competencies for the Specialized Oncology Nurse (CANO/ACIO). Retrieved from http://www.cano-acio.ca

Canadian Cancer Society's Advisory Committee on Cancer Statistics. (2015). Canadian Cancer Statistic 2015. Toronto, ON: Canadian Cancer Society. [May 2015, adapted June 2015] ISSN 0835-2976

Chelf, J.H., Agre, P., Axelrod, A., Cheney, L., Cole, D.D., Conrad, K., $\&$ Weaver, C. (2001). Cancer-related patient education: An overview of the last decade of evaluation and research. Oncology Nursing Forum, 28(7), 1139-1147.

Dalby, C.K., Nesbitt, M., Frechette, C.A., Kennerley, K., Lacoursiere, L.H., \& Buswell, L. (2013). Standardization of initial chemotherapy teaching to improve care. Clinical Journal of Oncology Nursing, 17(5), 472-475. doi:10.1188/13.CJON.472-475

D'Amato, S. (2008). Improving patient adherence with oral chemotherapy. Oncology Issues, 42-45.

Doak, C.C., Doak, L.G., \& Root, J.H. (1996). Teaching patients with low literacy skills. Philadelphia: J.B. Lippincott Company.

Keller, J. (2006). Implementation of a prechemotherapy educational intervention for women newly diagnosed with breast cancer. Clinical Journal of Oncology Nursing, 10(1), 57-63. doi:10.1188/06. CJON.57-60

Malone, P. (2007). Implementation of a prechemotherapy educational intervention. Clinical Journal of Oncology Nursing, 11(5), 707-710. doi:10.1188/07.CJON.707-710

Mann, K. (2011). Education and health promotion for new patients with cancer: A quality improvement model. Clinical Journal of Oncology Nursing, 15(1), 55-61. doi:10.1188/11.CJON.55-61

\section{CONCLUSION}

The nurse-led gynecology oncology chemotherapy education class at our cancer centre provided a standardized educational approach for chemotherapy-naive patients and caregivers in the outpatient setting. This nurse-led initiative created a pathway to empower patients and caregivers with knowledge and self-confidence, thereby potentially decreasing anxiety during their cancer journey. The nurse-led chemotherapy class enables the nurse to meet the CANO practice standards regarding supportive and therapeutic relationships, management of cancer symptoms, treatment side effects, and teaching and coaching (CANO, 2006). Since the inception of the class, nurses have reported a reduction in the number of patient phone calls relating to chemotherapy. The establishment of such a class provides a platform for the development of other, disease site specific patient education programs that can be offered in a variety of ways.

McCaughan, E., \& McKenna, H. (2007). Never-ending making sense: Towards a substantive theory of the information-seeking behaviour of newly diagnosed cancer patients. Journal of Clinical Nursing, 16(11), 2096-2104. doi:10.1111/j.1365-2702.2006.01817x

Oncology Patient Information System (OPIS) at Princess Margaret Cancer Centre (2010). Toronto, Ontario.

Papadakos, J., Brecher, E., Seki, J., Lee, R., Stewart, J., Abdelmutti, N. (2010, October). Effect of the PMH 'Chemo Questions?' class on anxiety and self-efficacy in cancer patients and caregivers: A summative evaluation. International Cancer Patient Education Meeting San Diego, California.

Portz, D., \& Johnston, M.P. (2014). Implementation of an evidencebased education practice change for patients with cancer. Clinical Journal of Oncology Nursing, 18(5), 36-40. doi:10.1188/14.CJON. S2.36-40

Princess Margaret Cancer Registry (2016). New primary gynecological cancer cases seen at Princess Margaret 2001-2015. Princess Margaret Cancer Centre. Toronto, Ontario.

Schofield, P., Jefford, M., Carey, M., Thomson, K., Evans, M., Baravelli, C., \& Aranda, S. (2008). Preparing patients for threatening medical treatments: Effects of a chemotherapy educational DVD on anxiety, unmet needs, and selfefficacy. Supportive Care in Cancer, 16(1), 37-45. doi:10.1007/ s00520-007-0273-4

Sheldon, L.K., Swanson, S., Dolce, A., Marsh, K., \& Summers, J. (2008). Putting evidence into practice: Evidence-based interventions for anxiety. Clinical Journal of Oncology Nursing, 12(5), 789-797. doi:10.1188/08.cjon.789-797

Williams, S., \& Schreier, A. (2004). The effect of education in managing side effects in women receiving chemotherapy for treatment of breast cancer. Oncology Nursing Forum, 31(1),16-23. doi:10.1188/04.ONF.E16-E23 